\title{
Direct reconstruction of the two-dimensional pair distribution function in partially ordered systems with angular correlations
}

\author{
I. A. Zaluzhnyy, ${ }^{1,2}$ R. P. Kurta, ${ }^{3}$ A. P. Menushenkov, ${ }^{2}$ B. I. Ostrovskii, ${ }^{4,5, *}$ and I. A. Vartanyants ${ }^{1,2, \dagger}$ \\ ${ }^{1}$ Deutsches Elektronen-Synchrotron DESY, Notkestraße 85, D-22607 Hamburg, Germany \\ ${ }^{2}$ National Research Nuclear University MEPhI (Moscow Engineering Physics Institute), Kashirskoe shosse 31, Moscow 115409, Russia \\ ${ }^{3}$ European XFEL GmbH, Holzkoppel 4, D-22869 Schenefeld, Germany \\ ${ }^{4}$ FSRC “Crystallography and Photonics,” Russian Academy of Sciences, Leninskii prospect 59, 119333 Moscow, Russia \\ ${ }^{5}$ Landau Institute for Theoretical Physics, Russian Academy of Sciences, prospect akademika Semenova 1-A, Chernogolovka 142432, Russia
}

(Received 19 May 2016; published 14 September 2016)

\begin{abstract}
An x-ray scattering approach to determine the two-dimensional (2D) pair distribution function (PDF) in partially ordered 2D systems is proposed. We derive relations between the structure factor and PDF that enable quantitative studies of positional and bond-orientational (BO) order in real space. We apply this approach in the x-ray study of a liquid crystal (LC) film undergoing the smectic- $A$-hexatic- $B$ phase transition, to analyze the interplay between the positional and $\mathrm{BO}$ order during the temperature evolution of the LC film. We analyze the positional correlation length in different directions in real space.
\end{abstract}

DOI: 10.1103/PhysRevE.94.030701

An absence of translational symmetry in disordered materials makes it challenging to characterize their structure and establish a structure-functional relationship [1-5]. Compared to crystalline matter, where a number of $\mathrm{x}$-ray, neutron, and electron scattering techniques are available for structural characterization, much less information is accessible in the experiments on disordered and partially ordered materials [6,7]. Despite the absence of long-range order present in crystals, disordered materials exhibit a number of structural features, such as short- and quasi-long-range order, bondorientational (BO) order, or dynamic heterogeneity, that can be also coupled to each other [8-11]. The development of reliable characterization techniques capable of revealing various types of structural order is an important task in materials research.

The local structure of a system can be conveniently described by the pair distribution function (PDF) $g(\mathbf{r})$, which defines the probability of finding a particle at the separation $\mathbf{r}$ from any other arbitrary chosen particle $[12,13]$. The angularaveraged PDF $g(r)$, called also the radial distribution function, is typically used to characterize the structure of liquids and amorphous solids [6,7]. The radial distribution function allows one to determine an average number of particles in a certain coordination shell and extract the positional correlation length. However, this one-dimensional (1D) function is not sensitive to orientational order in the system, which makes it difficult to use, for example, in the analysis of local atomic packing or BO order.

The methods of PDF reconstruction in higher dimensions can be found in Ref. [12] [see also Ref. [14], where a two-dimensional (2D) PDF was determined for the sample with cylindrical symmetry]. In this Rapid Communication we go beyond that and show that the $2 \mathrm{D}$ angular-resolved PDF $g(\mathbf{r})$ can be reconstructed directly from the measured diffraction patterns, which provides information on positional and orientational order in a partially ordered 2D system. We

\footnotetext{
*ostrenator@gmail.com

†ivan.vartaniants@desy.de
}

applied this approach in the x-ray study of a liquid crystal (LC) film undergoing the smectic- $A$-hexatic- $B$ phase transition [15], to analyze the interplay between the positional and BO order during the temperature evolution of the LC film.

Let us consider an x-ray scattering experiment on a homogeneous 2D system of $N$ identical particles, where the direction of the incoming x-ray beam is perpendicular to the sample plane. The structure factor $S(\mathbf{q})$ of such a system is related to the real-space PDF $g(\mathbf{r})$ via the Fourier transform [6,7]

$$
S(\mathbf{q})=I(\mathbf{q}) / N|f(\mathbf{q})|^{2}=1+\langle\rho\rangle \int(g(\mathbf{r})-1) e^{-i \mathbf{q} \cdot \mathbf{r}} d \mathbf{r},
$$

where $I(\mathbf{q})$ is the intensity measured in the forward scattering geometry at the momentum transfer vector $\mathbf{q},\langle\rho\rangle$ is an average density of the particles, and $|f(\mathbf{q})|$ is a form factor. One can decompose both $S(\mathbf{q})$ and $g(\mathbf{r})$ into the angular Fourier series

$$
S(q, \phi)=\sum_{n=-\infty}^{+\infty} S_{n}(q) e^{i n \phi}, \quad g(r, \theta)=\sum_{n=-\infty}^{+\infty} g_{n}(r) e^{i n \theta},
$$

where $\mathbf{q}=(q, \phi), \mathbf{r}=(r, \theta)$ are the polar coordinates, and $S_{n}(q), g_{n}(r)$ are Fourier coefficients (FCs) of $S(q, \phi)$ and $g(r, \theta)$, respectively. Then, by substituting Eqs. (2) into (1), one can find that FCs of the PDF $g_{n}(r)$ are related to FCs of the structure factor $S_{n}(q)$ via the Hankel transform [16]

$$
g_{n}(r)=\delta_{0, n}+\frac{1}{2 \pi\langle\rho\rangle i^{n}} \int_{0}^{+\infty}\left[S_{n}(q)-\delta_{0, n}\right] J_{n}(q r) q d q,
$$

where $\delta_{0, n}$ is the Kronecker delta and $J_{n}(q r)$ is the Bessel function of the first kind of integer order $n$. By substituting the FCs (3) into the Fourier series (2) one can readily determine the 2D PDF $g(\mathbf{r})$ in real space. If the form factor of the particles composing the system can be approximated to be isotropic $f(\mathbf{q})=f(q)$, then FCs of the structure factor $S_{n}(q)$ in Eq. (3) can be determined by using FCs of the scattered intensity $I_{n}(q), S_{n}(q)=I_{n}(q) /\left(N|f(q)|^{2}\right)$. The latter can be calculated either directly from the measured diffraction patterns, or 

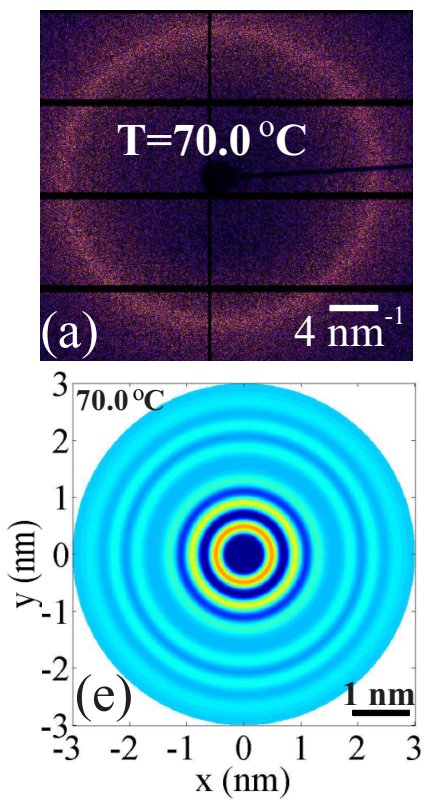
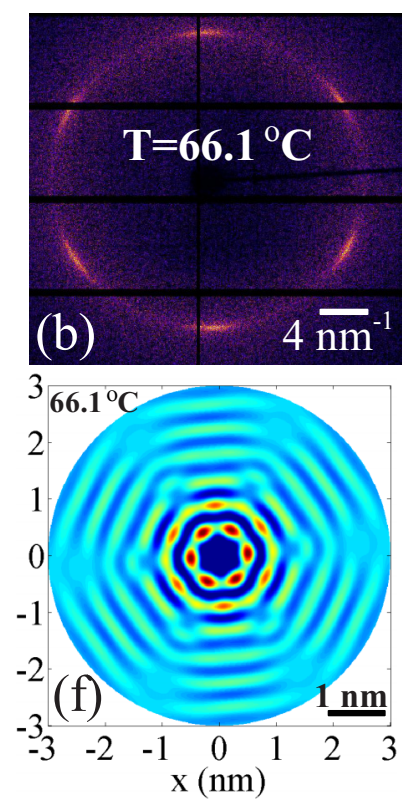
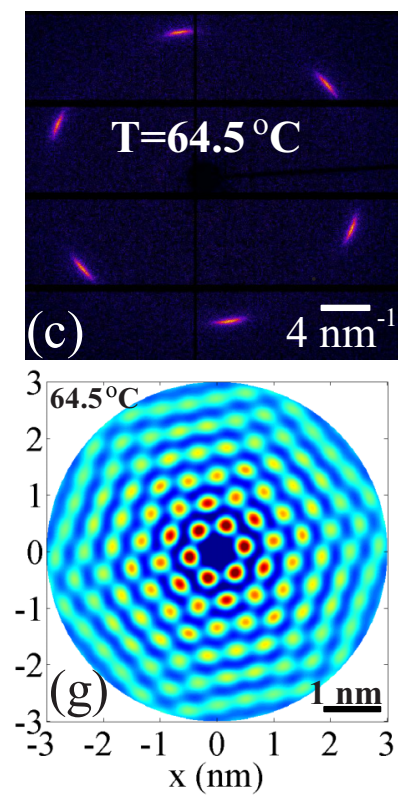
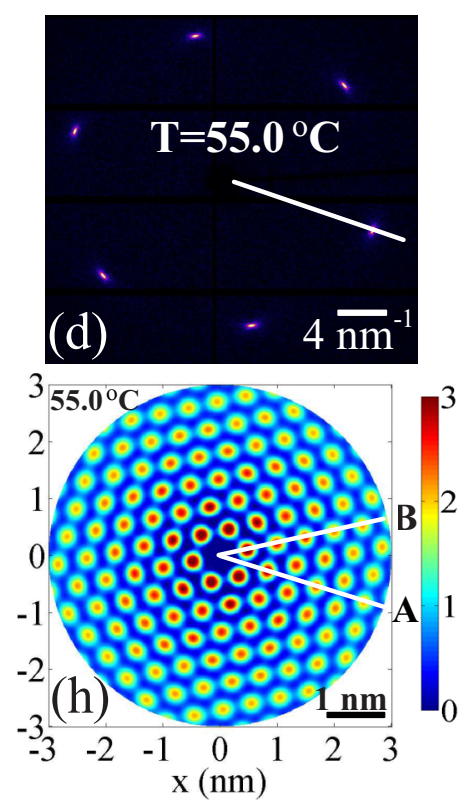

FIG. 1. (a)-(d) Diffraction patterns measured at different temperatures from the LC film undergoing the smectic- $A$-hexatic- $B$ phase transition. (e)-(h) The PDFs $g(\mathbf{r})$ determined from the diffraction patterns (a)-(d).

utilizing the x-ray cross-correlation analysis (XCCA) [17-21]. As it follows from this approach, the 2D PDF can be fully determined by the corresponding experimentally measured $2 \mathrm{D}$ intensity distribution.

In this Rapid Communication we applied the described approach to reconstruct the $2 \mathrm{D}$ PDF $g(\mathbf{r})$ in a LC film undergoing the smectic- $A$-hexatic- $B$ phase transition. An x-ray scattering experiment on a freely suspended LC film of the 3(10)OBC compound [22] was conducted at the Coherence Beamline P10 of the PETRA III synchrotron source at DESY with photons of energy $E=13 \mathrm{keV}$ (for experimental details, see Ref. [23]). The LC film consisted of about $2 \times 10^{3}$ layers that were formed by $\mathrm{LC}$ molecules oriented perpendicular to the layers. An incident $\mathrm{x}$-ray beam was perpendicular to the smectic- $A$ layers and the detector was positioned downstream in the transmission geometry. While decreasing the temperature of the LC film from $T=70.0^{\circ} \mathrm{C}$ to $T=55.0^{\circ} \mathrm{C}$ we observed the smectic- $A$-hexatic- $B$ phase transition at $T \approx 66.3{ }^{\circ} \mathrm{C}$ [23]. Typical diffraction patterns measured in the smectic- $A$ and hexatic- $B$ phases are shown in Figs. 1(a)-1(d). In the hightemperature smectic- $A$ phase the measured diffraction pattern consists of a broad ring [Fig. 1(a)], while at lower temperatures in the hexatic- $B$ phase the scattering ring splits into six arcs, following the appearance and development of the $\mathrm{BO}$ order [Figs. 1(b)-1(d)].

The 2D PDFs, describing the structure of a single LC layer [24], were determined at each temperature using Eqs. (2) and (3) and are shown in Figs. 1(e)-1(h). The integration in Eq. (3) was performed up to the maximum value $q_{\max }=1.9 \AA^{-1}$ accessible with the detector at the given experimental conditions [23] that determined the real-space resolution to be about $3.3 \AA$. First, the form factor of a single $3(10) \mathrm{OBC}$ molecule oriented along the x-ray beam was calculated numerically for the experimental conditions. Then, due to the fast rotation of the LC molecules around their long axis [25], this form factor was averaged over all possible orientations of the molecule and can be considered to be isotropic within a layer. In the frame of this approach the FCs of the structure factor can be directly obtained from the FCs of the scattered intensity using relations (1) and (2) [26]. FCs of intensity were determined by XCCA method [23]. In the hexatic- $B$ phase of LCs due to the sixfold symmetry of the diffraction patterns only FCs $S_{n}(q)$ of the order $n=6 m(m=0,1,2, \ldots)$ have nonzero values.

At the highest temperature $T=70.0^{\circ} \mathrm{C}$ in the smectic$A$ phase the PDF represents a set of concentric circles [Fig. 1(e)] that corresponds to the isotropic spatial distribution of molecules without angular correlations, which is typical for liquids and amorphous materials [6]. The position of the first ring is $4.8 \AA$, which corresponds to the average intermolecular distance [8]. Slightly below the smectic- $A$-hexatic- $B$ phase transition at $T=66.1^{\circ} \mathrm{C}$ the central uniform ring in the PDF splits into six well-separated bright spots [Fig. 1(f)]. This sixfold angular modulation in the positions of the nearestneighbor molecules appears due to emerging $\mathrm{BO}$ order that induces angular anisotropy in the initially isotropic positional order. One can also see that isotropy in particle correlations is also broken in the higher coordination shells, where the concentric rings observed in the smectic- $A$ phase transform to hexagons implying the presence of the hexatic- $B$ phase. At lower temperatures [Figs. 1(g) and 1(h)] deeper in the hexatic- $B$ phase, the tendency observed near the smectic- $A$ hexatic- $B$ phase transition becomes more pronounced due to increasing BO order. At $T=55.0^{\circ} \mathrm{C}$ all molecular positions at small distances are well localized, and the PDF consists of separated sharp peaks [Fig. 1(h)]. However, one can notice that the peaks of the PDF become more blurry at large values of $r$. This is a manifestation of the thermal angular fluctuations typical for hexatic- $B$ films, which reveals itself at large distances. 


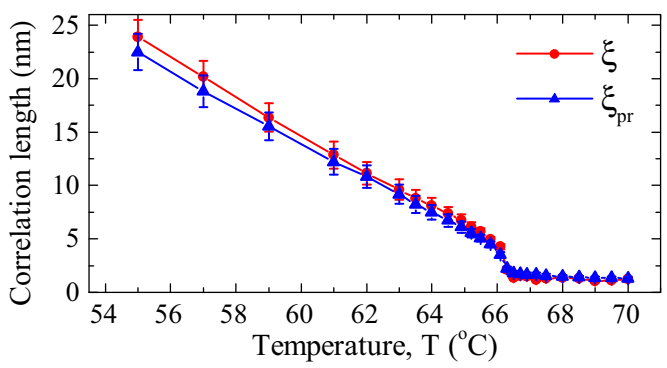

FIG. 2. Temperature dependence of the correlation length determined in different ways: $\xi$ from the radial section of scattered intensity (red circles) [23] and $\xi_{\text {pr }}$ from the projection of the PDF $g(\mathbf{r})$ onto the direction of the diffraction peak (blue triangles).

One of the key components of the structural analysis of a partially ordered system is the positional correlation length $\xi$, which is a basic quantity to determine a length scale over which the positional correlations between the particles in the system decay. Typically, it is evaluated as $\xi=1 / \gamma$, where $\gamma$ is the half width at half maximum (HWHM) of the diffraction peak in the radial direction [27]. In a previous work [23] we determined the correlation length $\xi$ from the radial section of intensity $I(q)$ through a single diffraction peak in the direction shown with the white line in Fig. 1(d). The temperature dependence of the positional correlation length $\xi$ determined in such a way is presented in Fig. 2. It can be readily shown, by applying the projection-slice theorem [28], that the same value of the correlation length can be also determined in real space from the projection of the function $g(\mathbf{r})-1$ on the direction of a diffraction peak that is specified with white line $A$ in Fig. 1(h). If the $x$ axis of Cartesian coordinates is chosen to be parallel to this direction, then the projection on this axis is [16]

$$
g_{\mathrm{pr}}(x)=\int_{-\infty}^{+\infty}[g(x, y)-1] d y=A \cos \left(q_{0} x\right) e^{-\gamma|x|}
$$

where $A=2 \pi \gamma /\langle\rho\rangle$. The parameter $\gamma$ could be extracted from the exponential fit of the envelope function of the projected PDF $g_{\mathrm{pr}}(x)$.

The projection of the PDF $g_{\mathrm{pr}}(x)$ on the direction $A$ is shown for different temperatures in Fig. 3. As it is expected, $g_{\mathrm{pr}}(x)$ is an oscillating function of distance with exponentially decreasing magnitude. Since the peak position $q_{0}$ almost does not depend on temperature, the period of oscillations is the same for both the hexatic- $B$ and smectic- $A$ phases. We determined the correlation length $\xi_{\mathrm{pr}}=1 / \gamma$ from the fit of the envelope function of $g_{\mathrm{pr}}(x)$ in the form of an exponent $A \exp (-\gamma x)$ [see Eq. (4)]. The obtained values of $\xi_{\text {pr }}$ as a function of temperature are shown in Fig. 2. They are in a good agreement with the values of $\xi$ obtained from the radial scans of intensity $I(q)$. The projection of the PDF on the direction between the diffraction peaks [direction $B$ in Fig. 1(h)] is equal to zero in accordance with the projection-slice theorem, since there is no measured scattered signal in this direction. We would like to note, if second-order diffraction peaks could be measured in this direction, this would lead to nonzero values of the corresponding PDF $g_{\mathrm{pr}}(x)$.

Clearly, the availability of the 2D PDF $g(\mathbf{r})$ shown in Figs. 1(e)-1(h) allows us to analyze the intermolecular
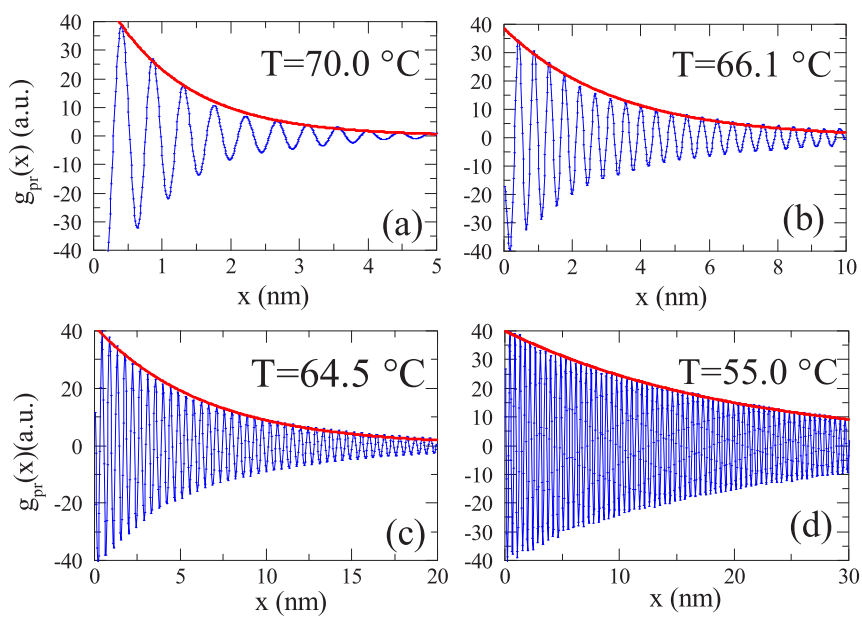

FIG. 3. Projection of the PDF $g_{\mathrm{pr}}(x)$ on the direction of the diffraction peak $A$ [see Fig. 1(h)] at different temperatures. The projection $g_{\mathrm{pr}}(x)$ is shown with the blue line and the envelope function in the form of an exponent $A \exp (-\gamma x)$ is shown with the red line.

correlations in 2D real space in different directions. As an example, the radial sections of the PDF in the directions $A$ and $B$, where index $A$ corresponds to the direction through the diffraction peaks and index $B$ between the diffraction peaks [see Fig. 1(h)] at two different temperatures, are shown in Fig. 4 [29]. Surprisingly, in the hexatic- $B$ phase the PDF decays faster with a distance in the direction between the diffraction peaks [see the insets in Figs. 4(a) and 4(b)]. We fitted the radial sections of the PDF shown in Fig. 4 with an envelope of the form $1+B r^{-0.5} \exp (-\gamma r)$, where $B$ and $\gamma$ were the fitting parameters. From these fits we determined the values of the positional correlation length $\xi_{A, B}=1 / \gamma_{A, B}$ in two different directions $A$ and $B$ shown in Fig. 1(h). At the lowest measured temperature $T=55.0^{\circ} \mathrm{C}$ in the hexatic- $B$ phase we obtained values $\xi_{A}=17 \mathrm{~nm}$ and $\xi_{B}=13 \mathrm{~nm}$ as compared to the one $\xi_{\mathrm{pr}}=23 \mathrm{~nm}$ obtained from the projection-slice theorem. We see from these results that the values of the correlation length strongly depend on the direction and are significantly lower than determined by the projection-slice theorem. To understand this behavior we performed an analysis in the frame of a general model described below.
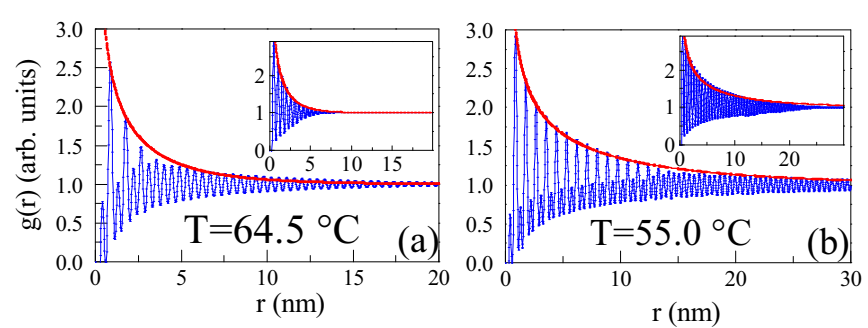

FIG. 4. The radial section of the PDF $g(\mathbf{r})$ in the direction of the diffraction peak $A$ [see Fig. 1(h)] at different temperatures. In the insets the radial section of $g(\mathbf{r})$ in the direction $B$ between the diffraction peaks [see Fig. 1(h)] is shown at the same temperature. The envelope function of the form $1+B r^{-0.5} \exp (-\gamma r)$, which was used for calculation of the correlation lengths $\xi_{1,2}=1 / \gamma_{1,2}$, is shown with the red line. 
We assume in the following that the structure factor of a monodomain system can be represented as a product of two terms [30],

$$
S(q, \phi)=S(q) S(\phi),
$$

where $S(q)$ and $S(\phi)$ correspond to the radial and angular dependence of the structure factor. It is common to use the Lorentzian function to describe the radial profile of the structure factor $S(q)$ in a partially ordered material, $S(q)=$ $\gamma^{2} /\left[\left(q-q_{0}\right)^{2}+\gamma^{2}\right]$, where $q_{0}$ and $\gamma$ define the position and HWHM of a characteristic peak in the scattered intensity distribution [27]. The angular part of the structure factor $S(\phi)$ can be quite generally represented as a Fourier series,

$$
S(\phi)=\sum_{n=-\infty}^{+\infty} C_{n} e^{i n \phi},
$$

where $C_{n}$ are FCs of $S(\phi)$, which can be defined to be real by an appropriate choice of the reference direction $\phi=0$. For a monodomain system considered here, $C_{n}$ with $n \neq 0$ are nothing but the generalized $\mathrm{BO}$ order parameters $[31,32]$. In the hexatic- $B$ phase of a liquid crystal only parameters of the order $n=6,12,18, \ldots$ can have nonzero values, due to sixfold rotational symmetry of the hexatic- $B$ structure [33]. With Eqs. (5) and (6) one can readily describe diffraction peaks in the hexatic- $B$ phase that are elongated in the azimuthal direction, as seen in Figs. 1(a)-1(d).

Using Eqs. (5) and (6) together with the general formula (3), the following expression for the PDF can be obtained [16],

$$
\begin{aligned}
g(r, \theta)= & +\frac{1}{2 \pi\langle\rho\rangle} \sum_{n=-\infty}^{+\infty}(-i)^{n} C_{n} e^{i n \theta} \\
& \times \int_{0}^{+\infty}\left[S(q)-\delta_{0, n}\right] J_{n}(q r) q d q .
\end{aligned}
$$

It is interesting to note here that in Eq. (5) for the structure factor the radial $q$ dependence and angular dependence were decoupled. At the same time the derived PDF in Eq. (7) shows strong coupling between the positional and $\mathrm{BO}$ order. This situation is typical for hexatic- $B$ liquid crystals where the coupling between the density fluctuations and hexatic- $B$ order parameters plays an important role [8]. As a result, both positional and BO order contribute to the PDF $g(r, \theta)$.

For large values of $r\left(q_{0} r \gg n^{2}\right)$ and small values of $\gamma$ $\left(\gamma \ll q_{0}\right)$ Eq. (7) can be simplified to [16]

$$
g(r, \theta) \rightarrow 1+B \frac{e^{-\gamma r}}{\sqrt{r}} \cos \left(q_{0} r-\frac{\pi}{4}\right) \sum_{n=-\infty}^{+\infty} C_{n} e^{i n \theta},
$$

where $B=\gamma /\langle\rho\rangle \sqrt{q_{0} / 2 \pi}$. At large distances the PDF $g(\mathbf{r})$ exponentially decays and approaches unity due to an absence of any correlations at large values of $r$. We note also that in asymptotic expression (8) the positional and BO order are decoupled. The positional order is described by an exponentially decaying oscillating term and the BO order coincides with the angular dependence of the structure factor (6). In the hexatic- $B$ phase the angular part of the structure factor $S(\phi)$ has a maximum in the direction of the peaks' maximum and approaches zero between the diffraction peaks. As such, the oscillations of the PDF $g(r, \theta)$ are suppressed in these directions (Fig. 4). Our analysis shows that in the frame of our model even asymptotically the positional correlation length can show apparently different values in different directions.

The situation will be different if measurements of the higher-order diffraction peaks were possible. In this case the angular anisotropy of the PDF at large distances would disappear and the values of the correlation length would converge. Our analysis showed that the values of the correlation length in different directions will be also the same in the case of symmetrical Bragg peaks, i.e., not elongated in the azimuthal direction.

In summary, we proposed an approach for the determination of the 2D pair distribution function in partially ordered $2 \mathrm{D}$ systems of identical particles with angular correlations directly from the experimentally measured x-ray diffraction patterns. The derived relations between the structure factor and 2D PDF have been used to reconstruct the PDF in hexatic- $B$ and smectic- $A$ films of liquid crystals. Application of the projection-slice theorem allowed us to determine the values of the correlation length in systems at different temperatures. The deduced PDF data clearly indicate both the exponential decay characteristic of the short-range positional in-plane order in the hexatic- $B$ phase, and the long-range $\mathrm{BO}$ order in the arrangement of the maximums of the PDF on the 2D maps in real space. By introducing a model describing scattering data from hexatic- $B$ films we analyzed in detail the behavior of the 2D PDF and explained an apparent different decay of the positional correlation length in different directions.

We foresee that the proposed method of the 2D PDFs reconstruction can be widely used for the analysis of partially ordered systems such as polymer colloids, suspensions of biological molecules, block copolymers, and liquid crystals. Our approach is particularly advantageous for analysis of spatial anisotropies of interparticle correlations.

We acknowledge support of this project and discussions with E. Weckert. We are thankful to A. Zozulya and M. Sprung as well as to the whole team of Coherence Beamline P10 at synchrotron source PETRA III for their support during beamtime and to $\mathrm{S}$. Funari for a careful reading of the manuscript. This work was partially supported by the Virtual Institute VH-VI-403 of the Helmholtz Association. The work of I.A.Z. and B.I.O. was partially supported by the Russian Science Foundation (Grant No. 14-12-00475).
[1] J.-L. Barrat and J.-P. Hansen, Basic Concepts for Simple and Complex Liquids (Cambridge University Press, Cambridge, U.K., 2003).

[2] S. R. Elliott, Nature (London) 354, 445 (1991).
[3] Y. Q. Cheng and E. Ma, Prog. Mater. Sci. 56, 379 (2011).

[4] T. P. Fraccia, G. P. Smith, G. Zanchetta, E. Paraboschi, Y. Yi, D. M. Walba, G. Dieci, N. A. Clark, and T. Bellini, Nat. Commun. 6, 6424 (2015). 
[5] J. A. Sellberg, C. Huang, T. A. McQueen, N. D. Loh, H. Laksmono, D. Schlesinger, R. G. Sierra, D. Nordlund, C. Y. Hampton, D. Starodub, D. P. DePonte, M. Beye, C. Chen, A. V. Martin, A. Barty, K. T. Wikfeldt, T. M. Weiss, C. Caronna, J. Feldkamp, L. B. Skinner, M. M. Seibert, M. Messerschmidt, G. J. Williams, S. Boutet, L. G. M. Pettersson, M. J. Bogan, and A. Nilsson, Nature (London) 510, 381 (2014).

[6] J. Als-Nielsen and D. McMorrow, Elements of Modern X-Ray Physics, 2nd ed. (Wiley, Hoboken, NJ, 2011).

[7] P. Chaikin and T. Lubensky, Principles of Condensed Matter Physics (Cambridge University Press, Cambridge, U.K., 1995).

[8] W. H. de Jeu, B. I. Ostrovskii, and A. N. Shalaginov, Rev. Mod. Phys. 75, 181 (2003).

[9] T. Kawasaki, T. Araki, and H. Tanaka, Phys. Rev. Lett. 99, 215701 (2007).

[10] E. P. Bernard and W. Krauth, Phys. Rev. Lett. 107, 155704 (2011).

[11] S. C. Kapfer and W. Krauth, Phys. Rev. Lett. 114, 035702 (2015).

[12] T. Egami and S. J. L. Billinge, Underneath the Bragg Peaks: Structural Analysis of Complex Materials (Pergamon, New York, 2003).

[13] B. Wu, T. Iwashita, and T. Egami, Phys. Rev. E 91, 032301 (2015).

[14] Y. He, R.-z. Hu, T. Egami, S. J. Poon, and G. J. Shiflet, Phys. Rev. Lett. 70, 2411 (1993).

[15] R. Pindak, D. E. Moncton, S. C. Davey, and J. W. Goodby, Phys. Rev. Lett. 46, 1135 (1981).

[16] See Supplemental Material at http://link.aps.org/supplemental/ 10.1103/PhysRevE.94.030701 for the details of the derivation of Eq. (3), x-ray diffraction from a thick LC film, projection-slice theorem, and the derivation of Eqs. (7) and (8) .

[17] P. Wochner, C. Gutt, T. Autenrieth, T. Demmer, V. Bugaev, A. D. Ortiz, A. Duri, F. Zontone, G. Grübel, and H. Dosch, Proc. Natl. Acad. Sci. USA 106, 11511 (2009).

[18] M. Altarelli, R. P. Kurta, and I. A. Vartanyants, Phys. Rev. B 82, 104207 (2010).

[19] R. P. Kurta, M. Altarelli, E. Weckert, and I. A. Vartanyants, Phys. Rev. B 85, 184204 (2012).

[20] R. P. Kurta, M. Altarelli, and I. A. Vartanyants, Adv. Condens. Matter Phys. 2013, 959835 (2013).

[21] R. P. Kurta, B. I. Ostrovskii, A. Singer, O. Y. Gorobtsov, A. Shabalin, D. Dzhigaev, O. M. Yefanov, A. V. Zozulya, M. Sprung, and I. A. Vartanyants, Phys. Rev. E 88, 044501 (2013).
[22] C. C. Huang, G. Nounesis, R. Geer, J. W. Goodby, and D. Guillon, Phys. Rev. A 39, 3741 (1989).

[23] I. A. Zaluzhnyy, R. P. Kurta, E. A. Sulyanova, O. Y. Gorobtsov, A. G. Shabalin, A. V. Zozulya, A. P. Menushenkov, M. Sprung, B. I. Ostrovskii, and I. A. Vartanyants, Phys. Rev. E 91, 042506 (2015).

[24] The measured LC film (about $5 \mu \mathrm{m}$ thick) consists of a stack of many molecular layers and strictly speaking cannot be considered as a pure 2D system. However, one can show that for such a layered system, in the absence of positional correlations between the layers, the structure factor is the same as for a single layer [16].

[25] J. Seliger, V. Žagar, and R. Blinc, Phys. Rev. A 17, 1149 (1978).

[26] Since the number of molecules $N$ illuminated with an x-ray beam was unknown, the normalization coefficient for $S_{n}(q)$ has been chosen in such a way that the calculated PDF $g(\mathbf{r})$ approaches zero at small distances $r$.

[27] H. Stanley, Introduction to Phase Transitions and Critical Phenomena (Oxford University Press, Oxford, U.K., 1971).

[28] In 2D the projection-slice theorem states that the results of the following two calculations are equal: Take a 2D function $g(\mathbf{r})$, project it onto a one-dimensional line, and do a Fourier transform of that projection, or take the same function $g(\mathbf{r})$, do a $2 \mathrm{D}$ Fourier transform first, and then slice it through its origin, which is parallel to the projection line [34] (see also Ref. [16] for details).

[29] The PDF has negative values close to the point $r=0$, which should be considered as an artifact caused by the finite experimental resolution.

[30] Our previous studies [21,23] indicated that the representation of the hexatic structure factor with Eqs. (5) and (6) is an approximation, since it does not take into account the fact that different FCs of the structure factor have different HWHM in the radial direction.

[31] P. J. Steinhardt, D. R. Nelson, and M. Ronchetti, Phys. Rev. B 28, 784 (1983).

[32] J. D. Brock, R. J. Birgeneau, D. Litster, and A. Aharony, Contemp. Phys. 30, 321 (1989).

[33] J. D. Brock, A. Aharony, R. J. Birgeneau, K. W. Evans-Lutterodt, J. D. Litster, P. M. Horn, G. B. Stephenson, and A. R. Tajbakhsh, Phys. Rev. Lett. 57, 98 (1986).

[34] A. D. Poularikas, The Transforms and Applications Handbook, 2nd ed. (CRC, Boca Raton, FL, 2000). 